\title{
A reduced order model for nonlinear vibroacoustic problems
}

\author{
Youssef Gerges $^{1, \mathrm{a}}$, Mohamed Guedri ${ }^{2}$, Emeline Sadoulet-Reboul ${ }^{1}$, Morvan Ouisse ${ }^{1}$, and Noureddine Bouhaddi ${ }^{1}$ \\ 1 Institut FEMTO-ST - Département de Mécanique Appliquée - 24 Chemin de l'Epitaphe - 25000 Besancon - France \\ 2 Institut préparatoire aux études dingénieurs de Nabeul (IPEIN) - Groupe Dynamique des Structures - 8000 Mrezgua - \\ Nabeul - Tunisie
}

\begin{abstract}
This work is related to geometrical nonlinearities applied to thin plates coupled with fluid-filled domain. Model reduction is performed to reduce the computation time. Reduced order model (ROM) is issued from the uncoupled linear problem and enriched with residues to describe the nonlinear behavior and coupling effects. To show the efficiency of the proposed method, numerical simulations in the case of an elastic plate closing an acoustic cavity are presented.
\end{abstract}

\section{Introduction}

This paper presents a model reduction method for nonlinear internal vibroacoustic problems. The starting point choice of the formulation: structural displacement and acoustic pressure (u,p) are employed in this paper. The finite element method (FEM) leads to an unsymmetrical matrix system with large dimensions. The nonlinear behavior to evaluate the nonlinear force at each step. This leads to high computational times which are not compatible with optimization and robustness analyses. In this context, model reduction using projection bases is one of the ways to reduce the computaion costs.

\section{Formulation of nonlinear vibroacoustic problem: Application for the thin plates}

The vibroacoustic problem considered in this work is presented as follow: let us consider a fluid-filled domain $\Gamma_{f}$ coupled with a structure $\Gamma_{s}$ presenting large displacements. $\Gamma_{f s}$ is the coupling surface. The structure is submitted to volume and surface loads $f_{v}(t)$ and $f_{s}(t)$. We start by recalling the structural and fluid uncoupled problems formulation.

\subsection{Structural problem formulation}

We recall the formulation of thin plates problem in nonlinear dynamics. Detailed formulation can be found in several books as [1].

Let us consider a thin plate $\Omega_{s}$ characterized by the middle surface $\Gamma_{m}$ and the thickness $\mathrm{h}$ in the reference $\mathfrak{R}(x, y, z)$. Plane stress hypothesis using the Love-Kirchoff model dedicated to thin plates is considered. The displacements are thus established by the knowledge of the middle surface motion denoted $(u, v, w)$ :

\footnotetext{
${ }^{a}$ e-mail: youssef.gerges@edu.univ-fcomte.fr
}

$$
\boldsymbol{u}(x, y, z, t)=\left\{\begin{array}{c}
u(x, y, t) \\
v(x, y, t) \\
w(x, y, t)
\end{array}\right\}-z\left\{\begin{array}{c}
w,_{x}(x, y, t) \\
w, y, y, t) \\
0
\end{array}\right\} .
$$

$u$ and $v$ are the in-plane displacements and $w$ is the transverse displacement.

Small deformations and moderate rotations hypotheses are used to write the $2^{\text {nd }}$ order Green-Lagrange strain tensor in a vector form as follows:

$$
E=E^{m}-z K^{b}
$$

where $E^{m}$ represents the in-plane deformations and $E^{b}=$ $z K^{b}$ the transverse deformations due to the bending effect. The structural equation of motion is deduced from the virtual work principle written as:

$$
\delta \int_{t_{1}}^{t_{2}}(U+T) d t=0
$$

where, $U$ is the potential energy and $T$ is the kinetic energy. Introducing the material stiffness matrices $D^{m}$ and $D^{b}$ respectively dedicated to the membrane and bending effects, the weak formulation of the equation of motion is written as:

$$
\begin{array}{r}
\int_{\Gamma_{m}}\left(\delta E^{m}\right)^{T} D^{m} E^{m} d S+\int_{\Gamma_{m}}\left(\delta K^{b}\right)^{T} D^{b} K^{b} d S+ \\
\int_{\Omega_{s}} \rho_{s} \frac{\partial^{2} \boldsymbol{u}}{\partial t^{2}} \delta \boldsymbol{u} d \Omega-\delta \Pi_{\text {ext }}=0,
\end{array}
$$

where,

$$
\begin{aligned}
& -D^{m}=\frac{E h}{1-v^{2}}\left[\begin{array}{llc}
1 & v & 0 \\
v & 1 & 0 \\
0 & 0 & \frac{1-v}{2}
\end{array}\right] \\
& -D^{b}=\frac{h^{2}}{12} D^{m}
\end{aligned}
$$

with:

- $\int_{\Omega_{s}} \rho_{s} \frac{\partial^{2} u}{\partial t^{2}} \delta \boldsymbol{u} d \Omega$ : virtual work due to the inertia,

- $\delta \Pi_{\text {ext }}$ : virtual work due to the external loads. 


\subsection{Fluid problem formulation}

Let us consider a fluid filled domain $\Omega_{f}$ coupled with an elastic surface $\Gamma_{f s}$. The equilibrium state is given by the equation:

$$
\Delta p=\frac{1}{c^{2}} \frac{\partial^{2} p}{\partial t^{2}},
$$

where $c$ is the sound of speed in the fluid.

The boundary condition applied on the coupling area $\Gamma_{f s}$ corresponds to the sliding condition:

$$
-\frac{\partial p}{\partial \boldsymbol{n}}=\rho_{f} \frac{\partial^{2} \boldsymbol{u}}{\partial t^{2}}
$$

where $\rho_{f}$ is the density of the fluid and $\boldsymbol{n}$ the fluid outward normal.

By applying the Green formula, the variational formulation of the fluid can be written as [2]:

$\frac{1}{\rho_{f}} \int_{\Omega_{f}} \nabla p \nabla \delta p d \Omega+\frac{1}{\rho_{f} c^{2}} \int_{\Omega_{f}} \frac{\partial^{2} p}{\partial t^{2}} \delta p d \Omega+\int_{\Gamma_{f s}} \frac{\partial^{2} \boldsymbol{u}}{\partial t^{2}} \boldsymbol{n} \delta p d \Gamma=0$.

\subsection{Coupled problem formulation}

For the coupled problem, the coupling effect is introduced in (4) as a surface load applied on the coupling surface $\Gamma_{f s}$. We write the virtual work due to the external loads as:

$$
\delta \Pi_{e x t}=\int_{\Omega_{s}} f_{v} \delta \boldsymbol{u} d \Omega+\int_{\Gamma_{s}} f_{s} \delta \boldsymbol{u} d \Gamma+\int_{\Gamma_{f s}} p \boldsymbol{n} \delta \boldsymbol{u} d \Gamma .
$$

In a classical way, the finite element formulation of the equations (4) and (7) leads to the following matrix formulation:

$$
\left[\begin{array}{cc}
M_{s} & 0 \\
\rho_{f} C^{T} & M_{f}
\end{array}\right]\left[\begin{array}{l}
\ddot{U} \\
\ddot{P}
\end{array}\right]+\left[\begin{array}{cc}
K_{s}(\boldsymbol{U})-C \\
0 & K_{f}
\end{array}\right]\left[\begin{array}{l}
\boldsymbol{U} \\
P
\end{array}\right]=\left[\begin{array}{l}
F \\
0
\end{array}\right]
$$

where:

- $M_{s}$ is the mass matrix of the structure. For thin plates problem $M_{s}=\left[\begin{array}{cc}M^{m} & 0 \\ 0 & M^{b}\end{array}\right]$ with $M^{m}$ and $M^{b}$ respectively the in-plane and bending mass matrices.

- $K_{s}$ is the stiffness matrix of the structure. $K_{s}$ is a function of $U$; for thin plates geometrical non-linearities it is the sum of a linear term arising from the linear problem $K_{s}^{l}=\left[\begin{array}{cc}K^{m} & 0 \\ 0 & K^{b}\end{array}\right]$ and a nonlinear term $\Delta K_{s}^{n l}(\boldsymbol{U})=$ $\left[\begin{array}{cc}0 & \frac{1}{2} C_{1}^{k} \\ C_{1}^{k} & C_{2}^{k}\end{array}\right]$ showing the coupling effect between the membrane and the bending parts of the matrix.

- $M_{f}$ and $K_{f}$ are the matrix respectively corresponding to the discretization of the kinematic energy and the compressibility of the fluid.

- $C$ is the coupling matrix corresponding to the action of the structure on the fluid or vice versa.

- $F$ is the structure excitation depending on time $t$.

The matrix system (9) can be expressed as a differential equation written as:

$$
M \ddot{X}+K(X) X=f(t) .
$$

If $f(t)$ is harmonic, one of the efficient techniques to solve the problem is the harmonic balance method [4]. The use of an arbitrary excitation requires the implementation of time integration methods [3]. In both cases, solving the problem is time consuming. In the next section, a reduced order method dedicated to this kind of problem is presented. The projecting basis has to take into account both nonlinear and coupling effects.

\section{Reduced order method}

The aim goal is to project the vector $X$ on a basis $T$ with small dimension.

$$
X(t)=T q(t) .
$$

The starting point is the classical approach based on the creation of the reduced Ritz sub-bases issued from the uncoupled problems. Nonlinear and coupling effects are considered as a perturbation of the non-coupled problems. The enrichment of the sub-bases with residues issued from these perturbations leads to a robust reduced basis dedicated to vibroacoustic problems. We start by introducing the reduced order method dedicated for the structural problem.

\subsection{ROM for nonlinear structural problem}

Let us consider the finite element formulation of a thin plate in vacuum. The matrix equation is written as follow:

$$
\left[\begin{array}{cc}
M^{m} & 0 \\
0 & M^{b}
\end{array}\right]\left[\begin{array}{c}
\ddot{U} \\
\ddot{W}
\end{array}\right]+\left[\begin{array}{cc}
K^{m} & \frac{1}{2} C_{1}^{k} \\
C_{1}^{k} & K^{b}+C_{2}^{k}
\end{array}\right]\left[\begin{array}{c}
U \\
W
\end{array}\right]=\left[\begin{array}{c}
F \\
0
\end{array}\right]
$$

Because of the coupling matrix, the high-frequencies inplane modes have to be included in the reduced basis so that the basis vectors $T_{s 0}$ have to verify the eigenvalue problem:

$$
\left\{\begin{array}{l}
\left(K^{m}-\omega^{2} M^{m}\right) \Phi^{m}=0, \\
\left(K^{b}-\omega^{2} M^{b}\right) \Phi^{b}=0 .
\end{array}\right.
$$

$\Phi^{m}$ and $\Phi^{b}$ are the membrane and bending eigenvectors of the uncoupled structural problem, and:

$$
T_{s 0}=\left[\begin{array}{cc}
\Phi^{m} & 0 \\
0 & \Phi^{b}
\end{array}\right]
$$

This basis usually leads to an accurate first estimation of the structure response. But for reanalysis studies and important nonlinear behavior, its enrichment is necessary to ensure robust analysis. In the next section we present the combined approximation (CA) method adapted to this kind of problem.

\section{Combined approximation method}

The combined approximation method is a method developed for reanalysis strategies. It consists on expanding each eigenvector of the initial problem in a binomial series based 
on the modified problem. Sub-bases developed are then combined to build on an overall reduction basis. For nonlinear analysis, nonlinear behavior is considered as a modification of the linear behavior, this allows the build on of the basis. The combined approximation method is presented in reference [5].

Let us consider the $v^{t h}$ eigenvalue problem of the equation (12):

$$
\left(K_{s}^{l}+\Delta K_{s}^{n l}(\boldsymbol{U})\right) T_{s}^{v}=\left(\omega^{\nu}\right)^{2} M_{s} T_{s}^{v}
$$

Multiplying the equation (15) by $\left(K_{s}^{l}\right)^{-1}$ leads to:

$$
(I+B) T_{s}^{v}=r_{0}^{v}
$$

with,

$$
\left\{\begin{array}{l}
B=\left(K_{s}^{l}\right)^{-1} \Delta K_{s}^{n l}(\boldsymbol{U}), \\
r_{0}^{v}=\left(\omega^{v}\right)^{2}\left(K_{s}^{l}\right)^{-1} M_{s} T_{s}^{v} \approx\left(\omega^{v}\right)^{2}\left(K_{s}^{l}\right)^{-1} M_{s} T_{s 0}^{v} .
\end{array}\right.
$$

Expanding the term $(I+B)^{-1}$ of the equation (16) leads to the equation:

$$
T_{s}^{v}=\left(I-B+B^{2}-\cdots\right) r_{0}^{v} \Rightarrow\left\{\begin{array}{l}
r_{1}^{v}=\left(K_{s}^{l}\right)^{-1} M_{s} T_{s 0}^{v} \\
r_{i}^{v}=-B r_{i-1}^{v}
\end{array}\right.
$$

$i=2,3, \cdots s$ with $s$ the degree of the Taylor decomposition. We built a sub-basis $r^{v}$ for each eigenvector $T_{s 0}^{v}$ as:

$$
r^{v}=\left[\begin{array}{llllll}
r_{1}^{v} & r_{2}^{v} & \ldots & r_{i}^{v} & \ldots & r_{s}^{v}
\end{array}\right]
$$

The sub-bases are combined to form a global basis:

$$
T_{s}=\left[\begin{array}{llllll}
r^{1} & r^{2} & \ldots & r^{v} & \ldots & r^{n}
\end{array}\right],
$$

with $n$ the number of eigenvectors retained for truncation. A mass normalization and a singular value decomposition $(S V D)$ are needed for the basis $T_{S}$ to ensure good conditioning.

\subsection{ROM for coupled fluid problem}

The main goal of this section is to propose a modal synthesis method that can take into account coupling effects. The homogeneous matrix formulation associated to the Equation (9) in the frequency domain is written as:

$$
\left(\left[\begin{array}{cc}
K_{s}(\boldsymbol{U})-C \\
0 & K_{f}
\end{array}\right]-\omega^{2}\left[\begin{array}{cc}
M_{s} & 0 \\
\rho_{f} C^{T} & M_{f}
\end{array}\right]\right)\left[\begin{array}{l}
\boldsymbol{U} \\
P
\end{array}\right]=\left[\begin{array}{l}
0 \\
0
\end{array}\right] .
$$

The term $\rho_{f} C^{T} \boldsymbol{U}$ can be interpreted as an excitation of the fluid due to the structure. For the fluid part, we have :

$$
\left(K_{f}-\omega^{2} M_{f}\right) P=\omega^{2} \rho_{f} C^{T} \boldsymbol{U}
$$

This excitation is not known but it can be approximated by projecting the displacement on the structural basis introduced in the previous section (14) or (20). Updating the fluid basis by including this response leads to a new reduced basis. This new residual basis is written as [6]:

$$
\Delta T_{f s}=\left(K_{f}-\omega_{c}^{2} M_{f}\right)^{-1} C^{T} T_{s},
$$

and should be decomposed in singular value to ensure orthogonality.

$$
T_{f}=\left[T_{f 0} \mid \Delta T_{f s}\right] .
$$

Once the reduced basis has been established, the model reduction is performed as follows:

$$
X=\left[\begin{array}{l}
\boldsymbol{U} \\
P
\end{array}\right]=\left[\begin{array}{cc}
T_{s} & 0 \\
0 & T_{f}
\end{array}\right]\left[\begin{array}{l}
q_{s} \\
q_{f}
\end{array}\right]=T q
$$

Dynamic equilibrium becomes:

$$
M_{r} \ddot{q}+K_{r}(q) q=f_{r}(t),
$$

where:

- $M_{r}$ is the reduced mass matrix: $M_{r}=T^{T} M T$

- $K_{r}$ is the reduced stiffness matrix: $K_{r}=T^{T} K T$

- $f_{r}$ is the reduced force vector: $f_{r}=T^{T} f$

The equation (26) is solved with a numerical integration in the time domain using the Newmark algorithm.

\section{Prediction indicators}

Results comparison tools are based on statistic indicators associated to the structure and fluid responses in addition to the energy indicators that result from acoustic and kinetic energies.

\subsection{Temporal moments}

Temporal moments are used in transient responses in order to compare between different models. It is used to qualify the model response. The $i^{\text {th }}$ order of the temporal moment of a response $y(t)$ is defined as [7]:

$$
M_{i}=\int_{-\infty}^{+\infty}\left(t-t_{s}\right)^{i}(y(t))^{2} d t
$$

where $t_{s}$ represents the temporal shift and $i$ the moment index order.

In this work, the following temporal moment $M_{i}$ is used with $t_{s}=0$ and normalized as follows:

$$
\left\{\begin{array}{l}
E=M_{0}: \text { Energy }\left(m^{2} s\right) \\
T=\frac{M_{1}}{M_{0}}: \text { Central time (centroid) }(s) \\
D^{2}=\frac{M_{2}}{M_{0}}-\left(\frac{M_{1}}{M_{0}}\right)^{2}: \text { Root mean square duration }\left(s^{2}\right)
\end{array}\right.
$$

\subsection{Energy indicators}

The indicators that are used are the acoustic energy and the mean square velocity.

The discretized form of the acoustic energy is expressed as follows:

$$
E_{a}=\frac{1}{2 \rho_{f}} P K_{f} P+\frac{1}{2 \rho_{f}} \dot{P} M_{f} \dot{P} .
$$

The discretized form of the mean square normal velocity is defined as:

$$
{\overline{V_{n}}}^{2}=\frac{1}{\left|S_{s}\right|} V M_{v_{n}} V
$$

where $M_{v_{n}}$ comes from the discretization of $\int v_{n}^{2} d S$. 
Table 1. Temporal moments for structural displacements.

\begin{tabular}{llll}
\hline & $\mathrm{E}$ & $\mathrm{T}$ & $\mathrm{D}$ \\
\hline Full model & $0.7 \mathrm{E}-4$ & 0.1 & $4.5 \mathrm{E}-3$ \\
MB error & $-2.6 \%$ & $0.0 \%$ & $0.0 \%$ \\
MBFE error & $-2.3 \%$ & $0.0 \%$ & $0.0 \%$ \\
CAFE error & $0.0 \%$ & $0.0 \%$ & $0.0 \%$ \\
\hline
\end{tabular}

\section{Numerical illustration}

A parallelepipedic acoustic cavity fixed with a thin plate is modeled. Dissipation energy is introduced by modeling a proportional damping $D=\alpha K_{s}^{l}$. The response due to a sweep sine excitation in the frequency band of the interest is studied. The full model response is compared to the results obtained with several reduction bases. The reduced order methods used in these applications are the following:

- reduced model using the uncoupled modal basis of the system (Modal basis - MB);

- enrichment of the uncoupled fluid modal basis by the static response of the fluid due to the presence of the structure (MB + fluid residues - MBFE);

- enrichment of the uncoupled structural basis with the $\mathrm{CA}$ and enrichment of the fluid uncoupled basis by the static response of the fluid due to the presence of the structure (CAFE).

The temporal moments for a period of $1 s$ of the structural displacement and the acoustic pressure are repsectively presented in Tables 1 and 2 . The variation of the mean square velocity and the acoustic energy are presented in Figures 1 and 2. The displayed result shows that the uncoupled modal basis (MB) is not sufficent to accurately represent the response. The enrichment of the fluid modal basis with the static response of the fluid due to the presence of the structure (MBFE) reduces the error compared to the full model but it stills inadequate for strongly nonlinear problems $(W / h>1.3)$ and for reanalysis problems. The combined approximation method enriched with the fluid response (CAFE) seems to be adequate for strongly nonlinear problems, the main drawback is the variation of the degree for the Taylor series development from an application to another that tends to make the computation diverge. Recent works are on a criteria for this problem.

\section{Conclusion}

A reduced order method adapted to nonlinear vibro-acoustic problems is presented. It is required for geometrical nonlinear problems and it can be extended to other types of nonlinearities. It consists in enriching the uncoupled modal basis with residues taking into account non-linearity with the combined approximation method and coupling conditions with static residues. It was mentioned that associating conditions is very important to ensure convergence. An example in the temporal space has been presented. An application of the approach in the frequency domain is conceivable using the harmonic balance method adapted for geometric non-linearities.

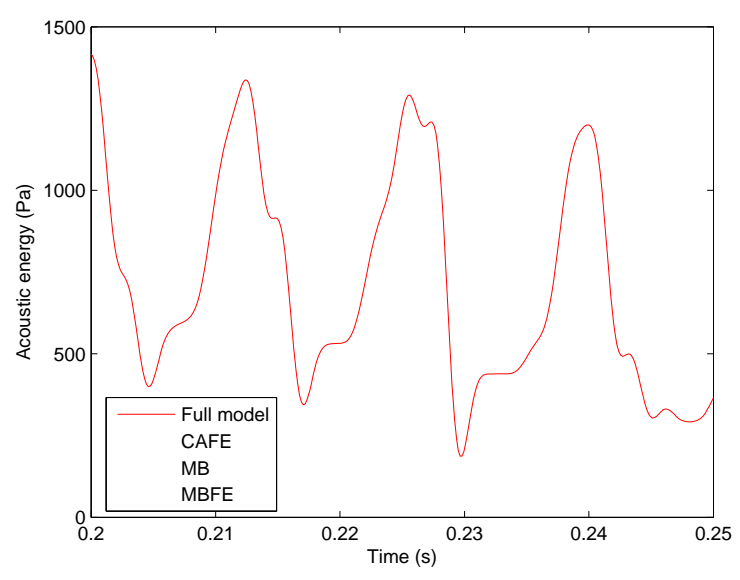

Fig. 1. Acoustic energy

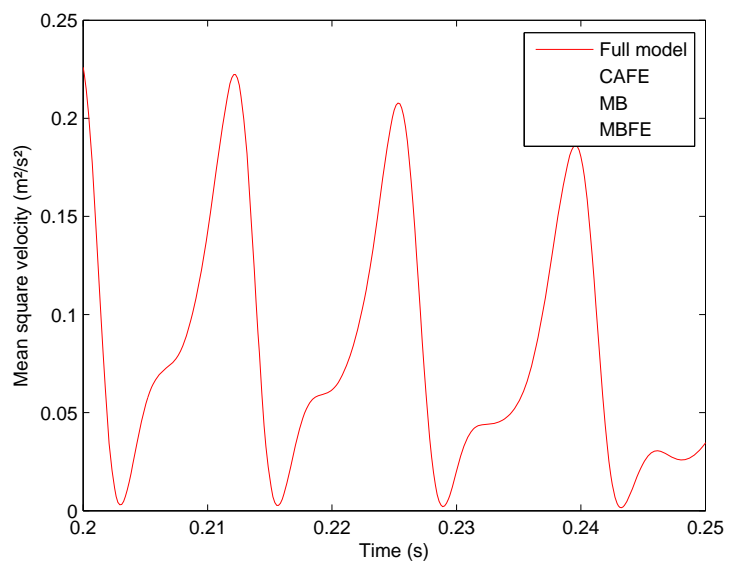

Fig. 2. Mean square velocity

Table 2. Temporal moments for acoustic pressure

\begin{tabular}{llll}
\hline & $\mathrm{E}$ & $\mathrm{T}$ & $\mathrm{D}$ \\
\hline Full model & $2.3 \mathrm{E} 7$ & 0.1 & $4.5 \mathrm{e}-3$ \\
MB error & $-1.8 \%$ & $-0.1 \%$ & $0.1 \%$ \\
MBFE error & $-1.6 \%$ & $0.0 \%$ & $0.1 \%$ \\
CAFE error & $0.0 \%$ & $0.0 \%$ & $0.0 \%$ \\
\hline
\end{tabular}

\section{References}

1. Zienkiewicz, O.C. and Taylor, R.L., The Finite Element Method (Fifth Edition, Volume 2: Solid Mechanics).

2. H. Morand and R. Ohayon, Interactions FluidesStructures (MASSON, 1992).

3. M. Géradin and D. Rixen, Mechanical Vibrations: Theory and Applications to Structural Dynamics (John Wiley \& Sons Ltd, 1997).

4. A. H. Nayfeh and D. T. Mook Nonlinear Oscillations (Wiley Classics Library, 1995).

5. U., Kirsch, M., Bogomolni and Sheinman, I., Comput. Methods Appl. Mech. Engrg 195, (2006) 4420-4432.

6. Q. H., Tran, M., Ouisse M. and N. Bouhaddi, Mechanical Systems and Signal Processing, 24, (2010) 164-181.

7. D. O. Smallwood, Prodceedings fo the 63rd Shock and Vibration Symposium, (October 1992) 389-399. 